\title{
Syntaktische Beiträge zur Geschichte der finnisch-ugrischen Konjunktionen
}

Konjunktionen sind sog. bedeutungsleere oder Formwörter, sie haben nur Funktionen; sie stellen die Verbindung zwischen Satzelementen oder Sätzen her und konstituieren zugleich die betr. Syntagmen oder Sätze; einen Hinweis auf die Äusserungssituation enthalten sie nicht. ${ }^{1}$ Die fi. Temporalkonjunktion sitten kuin 'nachdem' bezeichnet zwar eine Zeitrelation, hat aber keinen Zeitstellenwert und enthält deshalb keinen Hinweis auf die Äusserungssituation.

Wenn in der Semantik die Entwicklung vom Konkreten zum Abstrakten als einziges Gesetz des Bedeutungswandels gilt, muss die Konjunktion im Ausgestaltungsprozess der Sprache ein spätes Produkt sein. Das wird durch die historische Sprachforschung bestätigt. ${ }^{2}$

Dieser Umstand lässt die Konjunktionen als besonders geeignetes Studienmaterial im Zusammenhang mit der Frage nach dem Verhältnis von Sprache und Denken erscheinen. Wenn die Konjunktion die logische Beziehung zwischen Einheiten der Äusserung ausdrückt, ist sie diejenige Wortart, die am unmittelbarsten den Denkprozess abspiegelt. Einen anschaulichen Hinweis hierauf liefert schon die Wortfolge. Die Sprache als an die Zeit gebundenes Tun ist auf das Nacheinander ihrer Elemente in der Rede angewiesen, und das Sprachdenken bestimmt die Reihenfolge. Bei der reinen, d.h. der kopulativen und disjunktiven Nebenordnung (in letzterer aber nur im Syntagma) können die Einheiten vom syntakti-

1 Vgl. W. P. Schmidt, Skizze einer allgemeinen Theorie der Wortarten. AkWiss. u. Lit Mainz (1970), S. 8 (262); 14 (268).

${ }^{2}$ Vgl. z.B. O. Behaghel, Deutsche Syntax III (Heidelb. 1928), 48; J. Berrár, Magyar történeti mondattan (Bp. 1957), 62ff.; E. N. Setälä, Suomen kielen lauseoppi ${ }^{14}$ (Hels. 1960), $131 \mathrm{ff.}$ 
schen Standpunkt aus beliebig aufeinander folgen: "Sie kaufte Äpfel und Birnen Birnen und Äpfel"; "Er zündete sich eine Zigarette an und trat ans Fenster (oder umgekehrt)"; "Willst du warme oder kalte Milch?» "Kommst du oder gehst du?" Aber schon beim adversativen Verhältnis liegt die Reihenfolge fest, offenbar weil das Entgegengestellte in Denken und Sprache auf etwas projiziert werden muss: »Ich rief, aber er hörte nicht». Dasselbe gilt für die fgr. Sprachen. Sätze mit einheimischen Wörtern wie fi. mutta, ung. de ebenso wie mit Lehnwörtern (LpSchw men, russ. no, a) stehen in der adversativen Periode immer an 2. Stelle.

Komplizierter und daher wahrscheinlich historisch ergiebiger ist die Satzstellung bei Unterordnung. ${ }^{1}$ Ich muss mich mit ein paar Beispielen begnügen. Oft ist nicht zu entscheiden, ob es sich um Neben- oder Unterordnung handelt; vgl. syrj. ${ }^{2}$ ivlais zerö, šondia-a »draussen regnet es, während die Sonne scheint» (so die Grammatik; oder: "d. S. scheint aber»). Nach seiner Stellung wirkt $a$ als hervorhebende Partikel wie mehrere syrj. Wörter, die heute als Konjunktionen zählen; das Lehnwort ist in einen innersprachlichen Entwicklungsprozess hineingezogen worden. Ebenso menō premirujtisni, bura udžala da wich habe eine Prämie bekommen, weil ich gut arbeite» (: "ich arbeite ja gut»). Folgen dürften auch immer die Sätze mit bittö »als ob» (Vergleich ohne Kenntnis des Verglichenen unverständlich), $m i j$ »dass», $\operatorname{med}(-i m)$ ) damit» (ursprünglich adhortative Partikel, keine Unterordnung). Ebenso im Russischen.

Liess sich die Nachstellung hier aus russischem Vorbild oder aus ursprünglicher Parataxe erklären, so findet sich bei den meisten Nebensatzkonjunktionen die in der Wortfolge übliche Opposition von Vor- und Nachstellung; hier bleibt der Forschung noch viel zu tun. ${ }^{3}$ Die Ähnlichkeit mit dem Russischen ist auch hier unverkennbar. Historisch besonders interessant

1 Da mir gründliche Nachprüfung meiner Beobachtungen noch nicht möglich war, müssen sie als vorläufig betrachtet werden.

${ }^{2}$ Die Belege, wenn nichts anderes gesagt, aus Sovr. komi jazyk I (Skr. 1955), $270 \mathrm{ff}$.

${ }^{3}$ Sovr. komi jaz. II (Skr. 1967) behandelt die Satzstellung nicht im Zusammenhang. 
sind die Relativsätze. Die Relativa sind im Fgr. aus Interrogativa hervorgegangen und zumeist noch mit ihnen identisch. Dadurch wird die merkwürdige Tatsache leichter verständlich, dass ein so abstraktes Verhältnis, wie es die Relativa andeuten, doch schon so regelmässig bezeichnet wird.

Diese Herkunft setzt Voranstellung des Relativsatzes voraus. Sie gilt im Syrj. da, wo ein "notwendiger Relativsatz" ein pronominales Hauptsatzglied inhaltlich bestimmt: "Was säst du? das erntest du" $>$ "was du säst, ...» (Sovr. I 200). Ebenso die nahestehenden vergleichenden Sätze mit »je - desto, wie (viel) - so(viel)» u.ä. (202f.; II 236). Im ganzen aber stehen heute im Syrj. die Nebensätze häufiger hinter als vor ihrem Hauptsatz; Möglichkeit der Nachstellung in Sovr. II ausdrücklich erwähnt vom Relativsatz S. 206, kor mals» 208, 213, mij »das, was» 210, Subjektsatz mit kö 216, Prädikatsatz 218, Modalsätze 233, weiterführender Relativsatz 237; aus den Beispielen als überwiegend zu erschliessen bei mij, med "so dass" 208, Finalsatz 211, 227, indir. Fragesatz 211f., Kausalsatz 225. Voranstellung auffallend häufig beim Temporalsatz 219ff., Konditionalsatz 228ff. (hier besonders bei allgemeinen Sätzen 229, wenn im Hauptsatz seki »dann» steht oder wenn der Hauptsatz optativische Färbung oder einen Gegensatz ausdrückt: 230), Konzessivsatz 230ff., im Ausdruck »je - desto» 236.

Die jeweils "andere" Satzstellung ist das merkmaltragende Glied, also besonders motiviert. Meine Vermutung, dass 1ı.a. Hervorhebung eine Rolle spielt, fand ich bei relativem mij "Was" (210) und beim Kausalsatz (226) bestätigt; selbst liatte ich sie angenommen bei indir. Frage $(211 ; 227)$, Prädikatsatz (218), Temporalsatz mit "solange" oder mit solchen Hauptsätzen, in denen "immer» steht (220f.) sowie bei Nebensätzen mit $d a$ : temporale 222, kausale 226, konzessive 232f.; vgl. noch pir žö )sogleich» 222 und die lokalen Nebensätze $224 \mathrm{f}$.

Dass hier mit ein paar pauschalen Erklärungen nicht auszukommen ist, liegt auf der Hand. Voranstellung dürfte da ursprünglich gewesen sein, wo man von direkten Fragen ausgehen kann, also vor allem bei Relativsätzen, auch den adverbialen. Die Parallele zum Sprachdenkprozess ist deutlich. Der Relativsatz konstatiert nicht wie der Hauptsatz sondern 
orientiert auf ihn hin: "wo die Flüsse zusammentreffen, liegt eine Stadt». Dass sie zusammentreffen, wird nicht behauptet, sondern vorausgesetzt; die Aussage gilt nur als Lokalisierung des Hauptsatz-Tatbestandes. Diese Form der Abhängigkeit gibt die frühe Sprache durch eine Frage wieder, die ja auch auf die Antwort hin formuliert ist und nur durch sie zur Feststellung werden kann. Die Ursprünglichkeit der Fragefunktion ergibt sich aus der Etymologie der Konjunktionen ( $m$ - und $k$-Stämme).

Auch in anderen Fällen darf man mit Umwandlung aus Hauptsätzen rechnen, z.B. bei den Nebensätzen mit da. Dies russische Lehnwort ist schon altpermisch (Bedentung: "und») in angereihter Frage belegt; ${ }^{1}$ dic Umdeutung konnte also noch relativ spät erfolgen. Dass $d a$ keine Nebensatzkonjunktion war, zeigt seine Stellung am Ende des Vordersatzes: gortas vois da, saldattö bidön göstitni koröni »als er nach Hause kam, luden den Soldaten alle zu Gast». Die Partikel setzt den Hauptsatz etwas vom Vorhergehenden ab und schliesst ihn dadurch fester mit dem folgenden zusammen. ${ }^{2}$ Die Absetzung bereitet die Unterordnung vor.

Überall verbreitet im Fgr. ist der Konditionalsatz. Soweit Etymologien vorliegen, sind Kasusformen (fi. jos, ung. ha, viell. tscher. gân) oder Partikeln (syrj. kö, wotj. ke) oder Lehnwörter (Lapp. < Fi., Mord. < Russ., Obugr. < Syrj. usw.) zu erkennen. Die einheimischen Bildungen lassen sich wohl am ehesten an Demonstrativ- und Interrogativstämme anschliessen. Im Perm. und Tscher. erweist wieder die Binnen- (oder End-)Stellıng den Partikelcharakter; ein weiterer Hinweis ist die noch heute häufige Voranstellung des Konditionalsatzes. ${ }^{3}$ Die beträchtlichen etymologischen Unterschiede deuten trotz

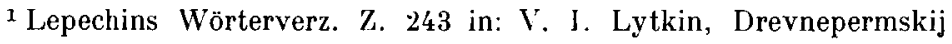
jazyk (M. 1952), 154.

${ }^{2} \mathrm{Vgl}$. die norddeutsche umgangssprachl. Erzählweise: „Er kam ža [= ja] denn nach Hause $\|$, und alle luden ihn ein.»

${ }^{3} \mathrm{Vgl}$. den dt. konjunktionslosen Konditionalsatz, der wie ein Fragesatz gebaut ist und immer vorangeht. - Auch tscher. gân "wenn" steht am Satzende, der Satz geht voran. - Brugmann, KVGr. S. 658 nimmt fürs Uridg. sogar generell Voranstellung des ungekennzeichneten Bedingungssatzes an.
} 
der weiten Verbreitung des Satztyps auf relativ späte, mühsame Erarbeitung. ${ }^{1}$ Jedenfalls ist der Entwicklungsweg länger als bei den Relativsätzen; darüber weiter unten.

Es gibt im Syrj. noch andere Spuren ehemaliger Parataxe. So kann nach Sätzen mit sömin, murtsa »kaum dass» der Nachsatz mit kid 'ź "wie» (vgl. russ. edva . ., kak, dt. "kaum . , als») beginnen. $^{2}$ Besondere Schwierigkeiten macht offenbar in vielen Sprachen die Wiedergabe des konzessiven Verhältnisses, so im Fgr. Am häufigsten hat es sich wohl aus dem konditionalen entwickelt - ist also jünger als dieses - aber auch aus parataktischen Konjunktionen oder Partikeln. Die konzessive Bedeutung steht oft neben mehreren anderen, und andererseits finden sich häufig mehrere Ausdrücke für konzessive Funktion. Vgl. fi. ehkä, vaikka, lahti (Eur. "vaikka"), -pa; ung. ${ }^{3}$ hol, holott, (erst 1508 mit konzess. Funktion belegt), mikor (a.O.S. 44f.), mig (1. konzess. Beleg 1832: MTESz 918), ha (a.O. II 7), noha, $h a-i s, h a-b a ́ r$ usw., zuweilen Imperativ statt Hypotaxe. Zur langen Entwicklungsgeschichte der ung. Konzessivsätze s. Simonyi a.O. S. 99, 127ff. Wog. N kwoss "wie, wenn auch" (kann auch weiter im Satz stehen); K kot'-kot' "sei es - sei es", kogs oder koti "obgleich", us "dennoch" (im Nachsatz nach unbezeichnetem Vordersatz); P kaš - kaš, T khoš »obgleich, wie immer». Cernecov und Rombandeeva sprechen in ihren grammatischen Skizzen nicht von Konjunktionen; sie seien wenig verbreitet. Syrj.: köt', köt med, med köt', daröm, da; einheimisch med "sei es». Der konzessive Satz kann vorangehen oder folgen; der übergeordnete Satz kann wie im Russischen mit Hauptsatzkonjunktionen eingeleitet werden; die Unterordnung ist also nicht vollständig. Wotj.. : ke no vobgleich", xot'a ... no ru. "chotja . . i», oźi ke no "abgesehen davon», ke no kann am Satzende stehen; oźi ke no kommt auch im Hauptsatz vor. Die Darstellung S. 326ff. zeigt auch anderwärts unvollständige Unterordnung, z.B. im temporalen Nebensatz: bere "nachdem", dirja

${ }^{1}$ Auch wenn hat sich gegen älteres $a b$ erst im 15. Jh. als allgemeine Konditionalkonjunktion durchgesetzt (Behaghel, a.O.S. 345ff.).

2 Sovr. komi jazyk II 222.

${ }^{3}$ Zs. Simonyi, A magyar kötőszók (Bp. 1883), 3.

${ }^{4}$ Gr. sovr. udm. jazyka I (Iževsk 1962), 331. 
"während" stehen nach dem Verb, sind also keine echten Konjunktionen gewesen. Konditionales $k e$ am Satzende, Nebensatz in den Beispielen voran. In einem Beispiel steht kausales no am Satzende, am Anfang des Nachsatzes soin; die Übersetzung lautet ". . . i potomu». Auch Sätze mit bere und dirja, die den Postpositionen nahestehen, gehen voran, während die übrigen Kausalsätze, z.B. mit ugoś, das man mit "nämlich» übersetzen könnte, regelmässig dem Hauptsatz folgen. Das ist ein Kennzeichen der Parataxe. Bei der Finalkonjunktion šuisa »damit» steht der Satz vor dem Hauptsatz - eine Nachwirkung der Gerundialkonstruktion; šu dien sind daneben noch ganz üblich. šu isa leitet auch Subjektund Objektsätze ein.

Aus den übrigen fgr. Sprachen konnte ich nur wenige Angaben zusammenstellen; in ihnen sind die Konjunktionen besonders schwach entwickelt. So etwa ostjs ${ }^{1} k u s ̌$ "wie, als, nachdem, kaum dass, obwohl»: Bedeutungsumfang weit, die konzessive Funktion nach allen Analogien sekundär. Im Gegensatz zu den entwickelteren fgr. Sprachen geht im Ostj. der Relativsatz fast immer voran, vertritt meist das Subj. oder Obj. und hat ein Stützwort im Hauptsatz. Die Nähe zum Fragesatz also noch deutlich. ${ }^{2}$ Tscher.: konzessiv koč, gânat "chotja", dial. dəkanät (< russ.). manân wie wotj. šuisa im Inhaltssatz, aber anscheinend nicht konzessiv. ${ }^{3}$ Mord.: E xot' "chotja", buti - to "jesli - to", kadik "pust"; M xot' wie E, katk wie E kadik. ${ }^{4}$ Voranstellung scheint weniger streng bedingt, doch lassen sich bei der starken Abhängigkeit vom Russischen nur schwer Schlüsse ziehen. ${ }^{5}$

Sieht man die Stellungsvielfalt der Nebensätze auf dem Hintergrund der grundsprachlichen Endstellung des Verbs, so kommt man zu einer klaren Zweiteilung: sprachliche Parataxe

\footnotetext{
1 D. R. Fuchs, LFSD (Bp. 1911), 165.

${ }^{2}$ Vgl. E. Vértes, Die ostjakischen Pronomina (Bp. 1966), $135 \mathrm{ff}$. J. Gulya, Ostyak Chrestomathy (Bloomington 1966), 85.

${ }^{3}$ Gr. mar. jaz, morf. I (J.-O. 1964), 179.

${ }^{4}$ M. N. Koljadenkov, Gr. mord. jaz. II (Sar. 1954), $303 \mathrm{f}$.

${ }^{5} \mathrm{Zu}$ den deutschen Konzessivkonjunktionen vgl. Behaghel, a.O.S. 51ff., $71,236,345,353,646 \mathrm{ff}$.
} 
ergab aus Gründen des Sprachdenkens Nachstellung des Hauptsatzes, Hypotaxe wegen Entstehung des Nebensatzes aus nominalen Satzteilen Voranstellung. Im heutigen Syrjänischen dagegen gilt im ganzen der Gegensatz ausnahmslose: überwiegende Nachstellung. Es müsste nun versucht werden zu ermitteln, wie regelmässig und wie lange der grundsprachliche Gegensatz gegolten hat, und welche Gründe für die Entstehung der heutigen Verteilung, die wohl auch in den übrigen fgr. Sprachen ohne kunstliterarische Tradition weithin herrscht, zu erkennen sind.

Lassen wir die relativ archaischen Konditional- und einige Typen der Relativsätze, die bereits kurz besprochen sind, beiseite, so ist wohl einer der Hauptgründe für die Zunahme der Nachstellung in der Satzwerdung adverbialer Bestimmungen zu sehen. Dabei ist jeweils festzustellen, ob die gedankliche und die syntaktische Entwicklung gleichen Schritt halten. Etwa mit heutigen fi. Mitteln: tultuaan kotiin $\sim$ kun hän tuli $k$, hän avasi ikkunan. Ähnliche Strukturen mögen nebeneinander gelegen haben. Die kürzere verbalnominale war Satzteil und konnte die Auffassung hervorrufen, dass der ursprüngliche Fragesatz, in dem die Unterordnung, wie oben geschildert, bereits angelegt war, nun zum »abhängigen Fragesatz» wurde. Es gehört jedoch zu den archaischen Zügen des Finnischen, dass hier die adverbialen Verbalnomina erhalten blieben, wenngleich ihre Bedeutung stark zurückgeht. Im Syrj. etwa konnten Ausdrücke wie "wegen des Heimkommens" unter Mitwirkung von potomu čto zu "deswegen, weil er heimkam" werden. Hierbei scheint n.a. die Länge des Nebensatzes eine Rolle gespielt zu haben: je komplexer die Mitteilung, desto länger der Satz, desto deutlicher gibt er einen selbständigen Tatbestand wieder, der sich nicht mehr als Satzteil noch als blosse Bestimmung auffassen lässt. Da er jedoch kontextlich trotz allem vom "Hauptsatz" bedingt ist, gliedert ihn das Sprachdenken nach dem bedingenden Satz ein: der Grund wird einsichtiger, wenn das zu Begründende bereits bekannt ist. Alles das sind Ursachen für die Nachstellung.

Ein interessanter Fall von Konjunktionswerdung (freilich unter türkischem Einfluss ${ }^{1}$ ) ist tscher. manân, wotj. šuisa

1 Vgl. N. I. Isanbaev, Trudy MarNII XV, S. 107. 
"sagend» in Sätzen wie: »er legte dem Pferd eine Decke über, es wird warm, sagend»; "ich meine, er kommt morgen, sagend". Semantisch ist die Befreiung aus der Redesituation erreicht; syntaktisch erfüllt "sagend" die Aufgabe einer Konjunktion; die Wortfolge hält aber den alten Satzteilcharakter noch fest. Isanbaev zeigt die schrittweise Loslösung aus der Redesituation; auch das ist zweifellos ein wichtiges Stadium in der Herausbildung des konjunktionalen Nebensatzes. In der archaischen Sprache haben, wie die heutige Umgangssprache noch zeigt, eingestreute Wort- und Satzfragen, Ausrufe u.a. den gleichmässigen Fluss der "Darstellung» viel häufiger unterbrochen als heute. Zeugnisse dafür sind die fgr. Konditionalund Relativsätze und die vielen endgestellten, partikelhaften, zunächst parataktischen Konjunktionen. In dem Masse nun, wie häufiger Gebrauch und zunehmende Länge der eingeschobenen Komplexe die ursprünglich gewiss dentlich vorschwebende Sprechsituation der sich aufdrängenden Frage verblassen liess zugunsten des übergreifenden Kontexts, der zu seiner Wiedergabe der gleichförmigen darstellenden Mitteilung bedurfte, desto mehr büssten alle diese Einschiebsel ihre alte Funktion und damit ihre kontextliche Sclbständigkeit ein: die Fragewörter wurden zu Konjunktionen, an die Stelle der alten asyndetischen, sprunghaften, aber erlebnisstarken Formulierung trat die Kennzeichnung des - vom Standpunkt der Information aus zweitrangigen - interpretierenden Nebenumstandes, der etwas zum Hauptgeschehen in irgend einer, von der Hauptlinie vablenkenden» Beziehung Stehendes angab. Für diese gedankliche Gliederung bedurfte die Sprache des "syntaktischen Gelenks", nicht mehr des subjektiv gebundenen "Ausdrucksworts".

Insofern gedankliche Gliederung "subjektiver" als blosse Mitteilung ist, steckt in jedem Nebensatz die Möglichkeit maffektischer» Voranstellung ebenso wie die der Nachstellung wegen "Achtergewicht" oder als Ausdruck gedanklicher Konsequenz aus dem Vorhergehenden. Wenn wir hier nach Spuren des Sprachdenkens suchen wollen, müssen wir uns weitgehend auf die Häufigkeit der Stellungstypen verlassen. Dafür fehlt es noch gänzlich an Unterlagen. Dass die Frage der Antwort vorangeht (korrelative oder das Subjekt umschreibende Relativsätze, Konditionalsätze, z.T. Konzessivsätze), entspricht ebenso dem 
zugrundeliegenden Sprachdenken wie Nachstellung etwa bei Kausal-, Konsekutiv-, weiterführenden ${ }^{\mathbf{1}}$ Relativssätzen (im Syrj. überhaupt bei den meisten Relativsatztypen ${ }^{2}$ ). Wenn wir finden, dass im Ostj. der (im ganzen seltene) Relativsatz meist vorangeht, so ist hier ein älteres Entwicklungsstadium festgehalten, das im Syrj. durch Anwachsen, Verselbständigung und Abrücken des Relativsatzes vom Fragesatz überwunden ist. Die allgemeine Entwicklung scheint also in Richtung auf die Nachstellung zu verlaufen.

Wo die Unterordnung nicht voll durchgeführt ist, hält sich anscheinend die Voranstellung länger. Hier ist der Ausländer auf die Darstellung der Handbücher angewiesen. Sovr. komi jaz. II übersetzt die oben genannten Sätze mit da am Ende mit russischen Nebensätzen, ohne auf einen Unterschied hinzuweisen. Da i.A. aber im Syrj. die Nebensatzkonjunktionen einen Satz einleiten, andererseits gerade da nicht "weil" u.ä. bedeutet hat, müssen wir von einer partikelhaften Bedeutung wie »ja» und Parataxe ausgehen. Später wurde z.B. das chronologische Nacheinander von Grund und Folge, in dem die Partikel auf das schon Bekannte hinwies, mehr im Sinne des gedanklichen Verhältnisses verstanden, ohne dass sich die sprachliche Form änderte.

Besonders einleuchtend ist die Parallelität von Sprachdenken und Satzstellung in Temporalperioden. Der vorzeitige Temporalsatz ist - zumindest in der Erzählliteratur - viel häufiger als der nachzeitige. Zu ihm sind auch Fälle zu rechnen wie das in Sovr. II 222 angeführte Beispiel "Als Paul ins Zelt trat, war Nina schon umgezogen». Die beiden Vorgänge sind gleichzeitig, aber der Zustand des Hauptsatzes reicht weiter in die Vergangenheit zurück. Wenn die ungegliederte parataktische Erzählung, in der das spätere Nebensatzereignis ohnehin voranging, zur hypotaktischen Periode wurde, bestand zunächst kein Anlass zur Umstellung. Mit der Gliederung der Ereigniskette befreite sich die Darstellung vom Zwang des Tatsachenablaufs, und nun konnte die Analogie anderer Nebensätze, das Streben

1 Vgl. Behaghel, a.O. III 771; Sovr. komi jaz. II 237.

2 A.O.S. 205 ff. 
nach über- oder unterdurchschnittlicher Bewertung u.a. die Stellung ändern. Auch Kontextgründe konnten einwirken; der Nebensatz musste z.B. folgen, wenn er das gleiche Subjekt wie der Hauptsatz hatte und dieses nicht im Nebensatz erscheinen sollte. Deutlich bewahrt den Platz der temporalen Bestimmung ein eingeschobener Satz wie "Jeder Vogel, sobald er flügge geworden ist, baut ein Nest». ${ }^{1}$ Nachstellung scheint aber zu gelten, wenn die Zeitangabe zum Hauptsatzverb, nicht zum ganzen Satz gehört. Auch in allgemeinen Wendungen wie "wenns regnet, wird es nass" wird wohl Nachstellung bevorzugt. Hier müssen Einzeluntersuchungen weiterhelfen.

Das oben skizzierte Bild von der Nebensatzstellung in den fgr. Sprachen führt zu einem Gedanken, der abschliessend mehr als Anregung vorgetragen als ausgebreitet werden soll. Wenn wir in den Unterschieden der Nebensatzstellung Spuren chronologischer Anordnung entdecken zu können glaubten, liesse sich diese These sinnvoll mit der Hypothese fortsetzen, dass auch in der Entstehung der einzelnen Satzverbindungen gewisse chronologische Unterschiede vorhanden seien. Vielleicht greifen diese Unterschiede mehr oder minder weit über die Grenzen der Einzelsprache hinaus, sofern die Parallelität zwischen Gebrauch des Nebensatzes und Sprachdenken besteht und nicht überwuchert wird.

Es war aufgefallen, dass der Relativsatz merkwürdig weit verbreitet ist, der Konzessivsatz aber selten vorkommt. Dazu stimmt, dass die Einleitung der Relativsätze durchweg aus einheimischem Material besteht, die des Konzessivsatzes meist entlehnt wurde. Weiter passt in dieses Bild, dass die Geschichte des fgr. Konzessivsatzes länger als die des Relativsatzes zu sein scheint. Wie angedeutet, setzen die nicht entlehnten Konzessivkonjunktionen i.A. bereits andere Nebensatzfunktionen (z.B. konditionale) voraus, der Relativsatz aber nur die direkte Frage. Schliesslich finden sich in der Stellung der Relativsatztypen mehr Reste archaischer Satzstellung als bei den Konzessivsätzen.

Ehe wir die Wichtigkeit des Relativsatzes für unsere Hypo-

1 Zu beiden Fällen a.O.S. 219. 
these noch etwas näher untersuchen, müssen wir nochmals kurz auf das Verhältnis von Para- und Hypotaxe eingehen. Es muss nämlich auffallen, dass parataktische Konjunktionen im ganzen mehr als hypotaktische entlehnt sind. So stammen alle koordinierenden Konjunktionen des Mordw. aus dem Russischen ${ }^{\mathbf{1}}$; alle bis auf den "und" entlehnt im Tscher.'; im Asyrj. 6 von 8, Syrj. $16-17$ von $18^{3}$, wotj. 11 von $15^{4}$. Die alten fgr. Schriftsprachen enthalten nur wenig Lehnkonjunktionen, die obugrischen Sprachen ebenfalls, sie sind überhaupt arm an dieser Wortart. Der Sinn solch eifriger Lehntätigkeit kann nicht darin liegen, dass die betr. Sprachen nicht imstande wären, Asyndese in Parataxe zu ïberführen; gerade in den obugr. Sprachen gibt es eine Reihe sogar korrelativer Konjunktionen. Der Grund muss wohl der sein, dass parataktische Konjunktionen in noch höherem Grade (und länger) als "überflüssig” erscheinen als hypotaktische. Verknüpfung kommt schon durch das pausenlose Nacheinander in der Äusserung zustande, auch bei Hypotaxe. Die Art der Verknüpfung ergibt sich aus dem Zusammenhang wie die der Hypotaxe. Aber das Faktum der Hypotaxe wird erst bei einer bestimmten Art rationaler Analyse deutlich, es ist eine höhere kognitive Leistung als die Parataxe. Nebenbei sei daran erinnert, dass attributive und syntagmatische Hypotaxe, wie gerade die uralischen und altaischen Sprachen zeigen, unabhängig von derjenigen der Sätze entstehen kann; es ist offenbar zweierlei, einen Tatbestand in sich und zwei Tatbestände untereinander zu gliedern. Innerhalb des Satzes kommt denn auch der Wortfolge kein Beweiswert für die Gedankenführung zu: das Attribut kann ebenso vor wie nach dem Leitwort stehen. Der Satz als faktische und situative Einheit ist vor der Äusserung angelegt, die Periode nicht.

Kann man also die Parataxe nur sprachpsychologisch für älter halten als die Hypotaxe, so möchte man etwa in den fgr.

\footnotetext{
1 Koljadenkov, a.O. I (Sar. 1962), 348.

${ }^{2}$ Istor. gr. mar. jaz., morf. I (J.-O. 1964), $176 \mathrm{ff}$.

${ }^{3}$ Sovr. komi jaz. I 271ff.

${ }^{4}$ Gr. sovr. udm. jaz. I (Iževsk 1962), $323 \mathrm{ff}$.
} 
Sprachen einige Typen des Relativsatzes und den expliziten Konzessivsatz für verschieden alt ansehen. Der Relativsatz ist hier aus dem Fragesatz entstanden, im Deutschen dagegen nur teilweise und spät ${ }^{\mathbf{1}}$, hauptsächlich aber aus Demonstrativa ${ }^{2}$. Der Weg von der Frage zum Relativsatz ist kürzer als über das Demonstrativ mit seinen Stufen ${ }^{3}$.

Macht man einen (freilich groben) Unterschied zwischen "semantischen" und "syntaktischen" Nebensätzen, so gehören Relativ- und indir. Fragesätze, in den perm. Sprachen auch die Konditionalsätze, zur 2. Gruppe ebenso wie die mit endgestelltem $d a$, während etwa med "damit» als echte Konjunktion Sätze der 1. Gruppe bildet. Es erscheint mir möglich, dass eine weitere Verfolgung dieses Gedankens zu dem Ergebnis führen könnte, dass Sätze der 2. Gruppe relativ-chronologisch im ganzen älter als die der 1. sind. Durch die Temporalsätze geht die Gruppierung mitten hindurch: Sätze mit "Relativkonjunktionen" gehören zur 2., solche mit "umschreibenden" wie "nachdem, während, bevor», die zeitliche Lage nicht zur Sprechsituation sondern nur in ihr angeben, zur 1. Gruppe. Sie sind häufig aus mehreren Wörtern gebildet, sind "motiviert" und schon deshalb jünger. Vornehmlich Sätze der 1. Gruppe werden ursprünglich nicht mit Konjunktionen sondern mit Gerundien wiedergegeben. Objektsätze scheinen eine Sonderstellung einzunehmen: obwohl zu Gruppe 2 gehörig, sind ihre Konjunktionen oft entlehnt oder fehlen ganz; hier scheint die Möglichkeit der Äusserung als direkte Rede die Entwicklung zurückgehalte!n zu haben.

W. SCHLACHTER

1 Behaghel, a.O. I 376.

${ }^{2}$ Ebda. III $711 \mathrm{ff}$. Das Gleiche soll nach Brugmann, KVGr. S. 664 im Uridg. geschehen sein, was mir freilich nicht sicher erwiesen zu sein scheint.

${ }^{3}$ K. Bühler, Sprachtheorie ${ }^{2}$ (Stuttg. 1965), $401 f$. 